\title{
Superoxide Dismutase and Catalase Activities in the Seminal Plasma of Normozoospermic and Asthenozoospermic Beagles
}

\author{
Eiichi KAWAKAMI ${ }^{1}$, Ariko TAKEMURA ${ }^{1)}$, Masaki SAKUMA ${ }^{1)}$, Mai TAKANO ${ }^{1)}$, Taichi HIRANO ${ }^{1)}$, \\ Tatsuya HORI ${ }^{1)}$ and Toshihiko TSUTSUI ${ }^{1)}$ \\ ${ }^{1)}$ Department of Reproduction, Nippon Veterinary and Life Science University, 1-7-1 Kyonan-cho, Musashino-shi, Tokyo 180-8602, \\ Japan
}

(Received 18 May 2006/Accepted 17 October 2006)

ABSTRACT. We measured the blood plasma testosterone (T) levels and superoxide dismutase (SOD) and catalase activities in the seminal plasma of the ejaculates of 5 normal (2-5 years old) and 5 asthenozoospermic (AZ-) (3-5 years old) Beagles. Sperm ejaculated by AZdogs was incubated for $3 \mathrm{hr}$ in Eagle's MEM only (controls) or Eagle's MEM containing $100 \mathrm{units} / \mathrm{ml}$ of SOD or catalase. Sperm motility was examined during incubation. The mean $( \pm \mathrm{SE})$ plasma T level of the AZ-dogs $(1.2 \pm 0.2 \mathrm{ng} / \mathrm{ml})$ was significantly lower than in the normal dogs $(2.5 \pm 0.2 \mathrm{ng} / \mathrm{m} l)(\mathrm{P}<0.005)$. The mean $( \pm \mathrm{SE})$ seminal plasma SOD and catalase activities $(18.8 \pm 1.9$ and $0.5 \pm 0.1$ unit/g protein, respectively) were significantly lower in the AZ-dogs than in the normal dog (43.3 \pm 2.5 and $2.2 \pm 0.4$ unit/g protein, respectively) $(\mathrm{P}<0.001$ and 0.01 , respectively). The motility of sperm incubated in Eagle's MEM containing SOD or catalase was significantly higher than that of control sperm incubated in only Eagle's MEM after 2 or $3 \mathrm{hr}$ of incubation $(\mathrm{P}<0.05)$. The results of this study indicate that poor $\mathrm{T}$ secretion by the testes and low antioxidant enzyme activities are related to $\mathrm{AZ}$ in the dog.

KEY WORDS: canine, catalase, seminal plasma, SOD.

Seminal plasma is known to contain reactive oxygen species (ROS) produced by testicular tissue [18] and sperm [2, 20], and elevated seminal plasma ROS concentrations in both humans $[1,16]$ and dogs [22] have been reported to be a cause of oligozoospermia, asthenozoospermia, and teratozoospermia. Low sperm motility and morphologically abnormal sperm occur as a result of sperm plasma membrane dysfunction caused by ROS [7].

Superoxide dismutase (SOD) [2, 6, 9] and catalase [6, 19, $24]$ are the main antioxidant enzymes in seminal plasma that prevent increases in ROS concentration in seminal plasma and protect the sperm against damage and oxidative stress caused by ROS. The SOD $[6,8,18]$ and catalase $[17,24]$ in seminal plasma are produced by the testis, epididymis, accessory reproductive organs, and sperm, and they are able to maintain sperm motility for a long time $[6,8]$. Seminal plasma SOD has been reported to have the same effect on canine sperm [5]. However, the cause of spermatogenic arrest in the dog is unknown [12]. In the present study, we examined the interaction between peripheral blood plasma testosterone $(\mathrm{T})$ levels and the activities of seminal plasma SOD and catalase in the dog. Based on the results of this study, we suggested some causes for spermatogenic dysfunction in the dog.

\section{MATERIALS AND METHODS}

Animals: Ten male Beagles aged 2-5 years were used in this study. They were cared for in our university and housed in pens with ample runs. Commercial dry dog food was provided twice a day, and the dogs were given free access to water. All animals were maintained according to the guidelines of the Animal Care and Use Committee of the Nippon
Veterinary and Life Science University.

The semen quality of the dogs was examined 3 times at one-week intervals. Five of the dogs (3-5 years old) were diagnosed with AZ based on their semen quality (percentage of actively motile sperm: less than $50 \%$ ).

Collection of ejaculated sperm and evaluation of semen quality: Semen specimens from the 5 normal and 5 AZ-Beagles were collected once weekly for 4 weeks by digital manipulation without a teaser bitch to measure, the SOD and catalase activities in the seminal plasma. Each semen specimen was examined for total semen volume, sperm concentration, and percentages of actively motile sperm and morphologically abnormal sperm by methods described previously [13]. Briefly, the sperm concentration in the semen was determined by hematocytometer counts, and the percentage of actively and progressively motile sperm on glass slides was estimated by examining 500 sperm using a warmplate and a light microscope. Sperm morphology was assessed after Rose Bengal staining ( $3 \mathrm{~g}$ of Rose Bengal and $1 \mathrm{~m} l$ of formalin in $99 \mathrm{~m} l$ distilled water).

Assay of SOD and catalase activities in seminal plasma: The sperm-rich fraction of the semen specimens collected from all dogs was centrifuged at $1,500 \times \mathrm{g}$ for $15 \mathrm{~min}$. The supernatant (seminal plasma) was collected, and the SOD and catalase activities in the seminal plasma were measured by enzyme analysis reactions using an SOD Assay Kit (Trevigen, Inc., MD, U.S.A.), Catalase Assay Kit (Cayman Chemical Company, MI, U.S.A.) and a spectrophotometer (UV-160 A, Shimazu Corporation, Tokyo, Japan) at an absorbance of $550 \mathrm{~nm}$ for both enzymes. Total protein concentrations in the seminal plasma were determined by the method of Bradford [4].

Blood sampling and blood plasma T assay: Heparinized 
peripheral blood samples were collected from superficial leg veins of all dogs on each day of semen collection. Blood samples were collected at 4 different times during the day (09:00, 12:00, 15:00, and 18:00) because of diurnal fluctuation of the plasma $\mathrm{T}$ levels in the $\operatorname{dog}[10,23]$. The plasma was then isolated and stored at $-20^{\circ} \mathrm{C}$ until assay.

Blood plasma $\mathrm{T}$ levels were measured by radioimmunoassay as described by Makino et al. [14]. Rabbit antiserum to $\mathrm{T}-11 \alpha$-succinate-BSA was used. The intra- and interassay coefficients of variation for $\mathrm{T}$ were $3.0 \%$ and $9.6 \%$, respectively.

Sperm incubation: The sperm ejaculated by the AZ-dogs was washed twice by centrifugation at $300 \times \mathrm{g}$ for $5 \mathrm{~min}$ in $5 \mathrm{~m} l$ of Eagle's minimal essential medium (MEM; Nissui Pharmaceutical Co., Ltd., Tokyo, Japan) supplemented with $1 \mathrm{mg} / \mathrm{ml} \mathrm{BSA}$ at $38^{\circ} \mathrm{C}$. The final sperm pellet was diluted to a concentration of $1 \times 10^{7} \mathrm{sperm} / \mathrm{m} l$ in MEM (control) and MEM supplemented with 100 units $/ \mathrm{m} l$ SOD derived from bovine erythrocytes (Calbiochem Inc., Darmstadt, Germany) or 100 units $/ \mathrm{m} l$ catalase derived from bovine liver (Wako Pure Chemical Industries Inc., Osaka, Japan). The sperm were incubated in loosely capped $120 \times 15 \mathrm{~mm}$ glass test tubes for $3 \mathrm{hr}$ at $38^{\circ} \mathrm{C}$ under an atmosphere of $5 \% \mathrm{CO}_{2}$ in air.

Statistical analysis: All semen and endocrine data were averaged for each dog, and the data for the normal and AZgroup, were summarized as mean values \pm standard error (SE). Differences between means were analyzed for statistical significance by Student's $t$ test. P values $<0.05$ were regarded as significant.

\section{RESULTS}

Semen quality and plasma T levels: The semen qualities of the normal and AZ-Beagles are shown in Table 1. The mean total number of sperm and mean percentages of actively motile sperm and morphologically abnormal sperm in semen ejaculated by the AZ-dogs were significantly lower than the mean values of the normal dogs $(\mathrm{P}<0.001)$. The very low sperm motility of the AZ-dogs was especially noteworthy.

The peripheral blood plasma $\mathrm{T}$ levels of the normal and AZ-dogs are shown in Table 1. The mean blood plasma T level of the AZ-dogs was significantly lower than in the normal dogs $(\mathrm{P}<0.005)$.

Seminal plasma SOD and catalase activities: The seminal plasma SOD and catalase activities of the normal and AZ-dogs are shown in Table 2. The mean SOD and catalase activities in the seminal plasma of the sperm-rich fraction of the AZ-dogs were significantly lower than the mean activities of the normal dogs ( $\mathrm{P}<0.001$ and 0.01 , respectively).

Sperm motility in the medium containing SOD or catalase: The changes in the mean percentages of actively motile sperm of the AZ-dogs in MEM containing SOD or catalase and in the control MEM during incubation are shown in Fig. 1. The mean percentages of motile sperm in MEM containing SOD and catalase were significantly
Table 1. Mean ( \pm SE) semen quality values and plasma testosterone levels in specimens collected once a week for four weeks from 5 normal (2-5 years old) and 5 asthenozoospermic (AZ-) (3-5 years old) Beagles

\begin{tabular}{lcc}
\hline & Normal dogs & AZ-dogs \\
\hline Total volume of semen $(\mathrm{m} l)$ & $11.2 \pm 4.2$ & $9.4 \pm 3.8$ \\
Total number of sperm $\left(\times 10^{6}\right)$ & $558.5 \pm 72.4$ & $127.3 \pm 47.2^{* *}$ \\
Actively motile sperm $(\%)$ & $82.3 \pm 9.1$ & $19.9 \pm 3.6^{* *}$ \\
Abnormaln sperm $(\%)$ & $3.6 \pm 1.5$ & $16.2 \pm 1.6^{* *}$ \\
Testosterone $(\mathrm{ng} / \mathrm{m} l)$ & $2.54 \pm 0.17$ & $1.22 \pm 0.24^{*}$
\end{tabular}

$* * \mathrm{P}<0.001$, compared with the normal dogs.

$* \mathrm{P}<0.005$, compared with the normal dogs.

Table 2. Mean $( \pm \mathrm{SE})$ superoxide dismutase (SOD) and catalase activities (units/g protein) in the seminal plasma of specimens collected once a week for four weeks from 5 normal and 5 asthenozoospermic (AZ-) Beagles

\begin{tabular}{lrc}
\hline & Normal dogs & \multicolumn{1}{c}{ AZ-dogs } \\
\hline SOD & $43.34 \pm 2.47$ & $18.82 \pm 1.85^{* *}$ \\
Catalase & $2.18 \pm 0.38$ & $0.48 \pm 0.11^{*}$ \\
\hline
\end{tabular}

$* * \mathrm{P}<0.001$, compared with the normal dogs.

* $\mathrm{P}<0.01$, compared with the normal dogs.

higher than for the controls in MEM only after 2 or $3 \mathrm{hr}$ of incubation $(\mathrm{P}<0.05)$.

\section{DISCUSSION}

SOD $[6,8]$ and catalase $[19,24]$ are very important antioxidant enzymes in seminal plasma $[9,11]$. An increase in the seminal plasma ROS concentration has been reported to cause poor semen quality in humans $[1,16]$ and dogs [22], and low SOD [2] and catalase [24] activities in seminal plasma cause an increase in the ROS concentration. Seminal plasma SOD has been found to protect canine sperm from damage by oxidative stress caused by ROS [5]. We assume that the low SOD and catalase activities in the seminal plasma of the AZ-dogs in the present study were closely related to the low motility of the ejaculated sperm. The seminal plasma ROS concentration of the AZ-dogs may be higher than in normozoospermic dogs.

The mean blood plasma $\mathrm{T}$ level of the AZ-dogs in this study was lower than in the normal dogs. As a result of the low $\mathrm{T}$ secretory function of canine testes, epididymal and prostatic function declines and abnormal seminal $\mathrm{pH}$ and osmotic pressure values develop [12]. The low motility of canine sperm is caused by abnormal $\mathrm{pH}$ and osmotic pressure of the seminal plasma [12]. Other factors responsible for low sperm motility are thought to be low SOD [21] and catalase [24] activities and high ROS concentrations [1, 3] in seminal plasma. Since the epididymis and prostate produce antioxidant enzymes [6, 15], epididymal and prostatic dysfunction caused by poor $\mathrm{T}$ secretion by the testes is 


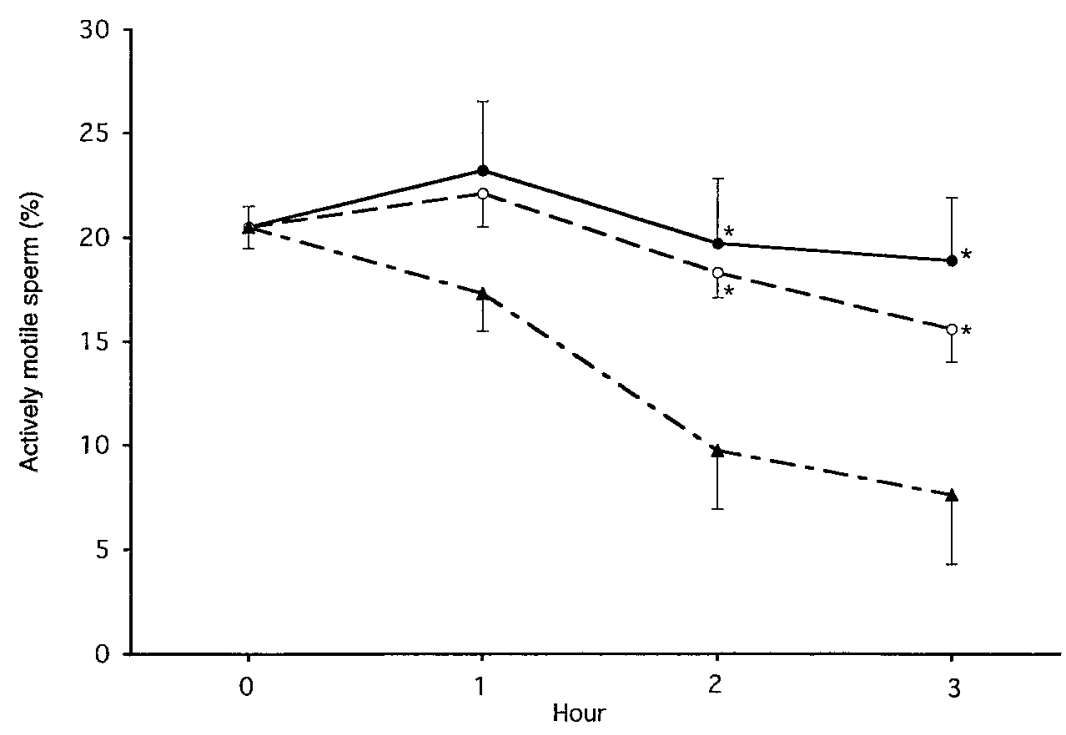

Fig. 1. Changes in the mean $( \pm \mathrm{SE})$ percentages of actively motile sperm in 5 asthenozoospermic dogs after 1,2, and $3 \mathrm{hr}$ of incubation in MEM with 100 units $/ \mathrm{m} l$ superoxide dismutase ( ) or 100 units $/ \mathrm{m} l$ catalase $(\bigcirc \cdots \bigcirc)$ and in MEM without the enzymes $(\mathbf{A}---\mathbf{A}) .{ }^{*} \mathrm{P}<0.05$, compared with MEM without the enzymes.

assumed to be responsible for the low SOD and catalase activities in the seminal plasma.

The results of the present study demonstrate that canine sperm motility can be maintained by the effects of SOD and catalase. Given that the mean seminal plasma SOD activity was higher than the catalase activity in the normal dogs and that the percentage of actively motile sperm was higher in MEM containing SOD than in MEM containing catalase, we believe that SOD is a more effective and important antioxidant enzyme than catalase for maintenance of canine sperm motility.

We therefore concluded that the low sperm motility of the AZ-dogs in this study was attributable to both low blood plasma T levels and low seminal plasma SOD and catalase activities.

\section{REFERENCES}

1. Aitken, R.J., Clarkson, J.S., Hargreave, T.B., Irvine, D.S. and Wu, F.C.W. 1989. Analysis of the relationship between defective sperm function and the generation of reactive oxygen species in cases of oligozoospermia. J. Androl. 10: 214-220.

2. Alkan, I., Simsek, F., Haklar, G., Kervancioglu, E., Ozveri, H., Yalcin, S. and Akdas, A. 1997. Reactive oxygen species production by the spermatozoa of patients with idiopathic infertility: relationship to seminal plasma antioxidants. J. Urol. 157: 140-143.

3. Baumber, J., Ball, B.A., Gravance, C.G., Medina, V. and Davies-Morel, M.C. 2000. The effect of reactive oxygen species on equine sperm motility, viability, acrosomal integrity, mitochondrial membrane potential, and membrane lipid perocidation. J. Androl. 21: 895-902.

4. Bradford, M. M. 1976. A rapid and sensitive method for the quantitation of microgram quantitie of protein utilizing the principle of protein-dye binding. Anal. Chem. 72: 248-254.

5. Cassani, P., Beconi, M.T. and O'flaherty, C. 2005. Relationship between total superoxide dismutase activity with lipid peroxidation, dynamics and morphological parameters in canine semen. Anim. Reprod. Sci. 86: 163-173.

6. Chen, H., Chow, P.H., Cheng, S.K., Cheung, A.L., Cheng, L.Y. and O, W.S. 2003. Male genital tract antioxidant enzymes: their source, function in the female, and ability to preserve sperm DNA integrity in the golden hamster. $J$. Androl. 24: 704-711.

7. Christova, Y., James, P.S. and Jones, R. 2004. Lipid diffusion in sperm plasma membranes exposed to peroxidative injury from oxygen free radicals. Mol. Reprod. Dev. 68: 365-372.

8. Foote, R.H., Brockett, C.C. and Kaproth, M.T. 2002. Motility and fertility of bull sperm in whole milk extender containing antioxidants. Anim. Reprod. Sci. 71: 13-23.

9. Gavella, M., Lipovac, V., Vucic, M. and Rocic, B. 1996. Relationship of sperm superoxide dismutase-like activity with other sperm-specific enzymes and experimentally induced lipid peroxidation in infertile men. Andrologia 28: 223-229.

10. Inaba, T., Shimizu, R. and Imori, T. 1977. Radioimmunoassay for male canine plasma 4-androstenedione, testosterone and 5 $\alpha$-dihydrotestosterone. Jpn. J. Anim. Reprod. 23: 63-71 (in Japanese with English summary).

11. Jeulin, C., Soufir, J.C., Weber, P., Laval-Martin, D. and Calvayrac, R. 1989. Catalase activity in human spermatozoa and seminal plasma. Gamate Res. 24: 185-196.

12. Kawakami, E., Hori, T. and Tsutsui, T. 1998. Changes in semen quality and in vitro sperm capacitation during various frequencies of semen collection in dogs with both asthenozoospermia and teratozoospermia. J. Vet. Med. Sci. 60: 607-614.

13. Kawakami, E., Tsutsui, T., Yamada, M. and Yamauchi, M. 1984. Cryptorchidism in the dog: Occureence of cryptorchidism and semen quality in the cryptorchid dog. Jpn. J. Vet. Sci. 46: 303-308.

14. Makino, T., Inano, K., Yoshida, T., Den, N., Takagi, S. and 
Kanbegawa, A. 1973. Radioimmunoassay of sex steroid hormone (4): Radioimmunoassay of testosterone. Clin. Endocrinol. (Tokyo) 21: 867-873.

15. Maxwell, W.M. and Stojanov, T. 1996. Liquid storage of ram semen in the absence or presence of some antioxidants. Reprod. Fertil. Dev. 8: 1013-1020.

16. Mazzilli, F., Ronconi, C., Rossi, T., Dondero, F. and Marchesini, M. 1994. Superoxide anion in human semen related to seminal parameteres and clinical aspects. Fertil. Steril. 62: 862-868.

17. Misro, M.M., Choudhury, L., Upreti, K., Gautam, D., Chaki, S.P., Mahajan, A.S. and Babbar, R. 2004. Use of hydrogen peroxide to assess the sperm susceptibility to oxidative stress in subjects presenting a normal semen profile. Int. J. Androl. 27: 82-87.

18. Peltola, V., Huhtaniemi, I. and Ahotupa, M. 1995. Abdominal position of the rat testis is associated with high level of lipid peroxidation. Biol. Reprod. 53: 1146-1150.

19. Rossi, T., Muzzilli, F., Delfino, M. and Dondero, F. 2001. Improved human sperm recovery using superoxide dismutase and catalase supplementation in semen cryopreservation procedure. Cell Tissue Bank. 2: 9-13.

20. Sharma, R.K. and Agarwal, A. 1996. Role of reactive oxygen species in male infertility. Urology 48: 835-850.

21. Siciliano, L., Tarantino, P., Longobardi, F., Rago, V., De Stefano, C. and Carpino, A. 2001. Impaired seminal antioxidant capacity in human semen with hyperviscosity or oligoasthenozoospermia. J. Androl. 22: 798-803.

22. Tselkas, K., Saratsis, P., Karagianidis, A. and Samouilidis, S. 2000. Extracellular presence of reactive oxygen species (ROS) in fresh and frozen-thawed canine semen and their effects on some semen parameters. Dtsch. Tierarztl. Wochenschr. 107: 69-72.

23. Tsutsui, T., Tsuji, J., Kawakami, E., Yamada, Y., Amano, T., Yamauchi, M. and Ogasa, A. 1987. Fluctuations in peripheral plasma androgen levels in peripubertal dogs. Jpn. J. Vet. Sci. 49: 751-755.

24. Zini, A. and Schlegel, P.N. 1996. Catalase mRNA expression in the male rat reproductive tract. J. Androl. 17: 473-480. 